\title{
Modeling of dielectric barrier discharge and resulting fluid dynamics
}

\author{
Balaji Jayaraman*1 ${ }^{1}$, Siddharth Thakur* $*^{2}$ and Wei Shyy**3 \\ *Dept. of Mechanical and Aerospace Engineering, \\ University of Florida, Gainesville, FL-32611 \\ **Department of Aerospace Engineering \\ University of Michigan, Ann Arbor, MI 48109
}

\begin{abstract}
Glow discharge at atmospheric pressure using a dielectric barrier discharge can induce fluid flow, and operate as an actuator in flow control. In the present work, a modeling framework is presented to study the evolution and interaction of such athermal non-equilibrium plasma discharges in conjunction with low Mach number fluid dynamics. Under atmospheric pressure, the discharge can be simulated using a plasmafluid instead of a kinetic model. The plasma and fluid species are treated as a two-fluid system coupled through force and pressure interactions, over a few decades of length and time scales. The multiple-scale processes such as convection, diffusion, and reaction/ionization mechanisms make the transport equations of the plasma dynamics stiff. To handle the stiffness, a sequential finite-volume operator-split algorithm capable of conserving space charge is employed. The fluid dynamics is solved using a pressurebased algorithm in a multi-block framework and can handle 3-D curvilinear grids. A body force treatment is devised to link the plasma dynamics and fluid dynamics. The resulting body force field can improve a previously proposed analytical-empirical treatment. The outcome of the body force field on the fluid flow is an attached wall jet type flow capable of modifying the near wall flow structures.
\end{abstract}

Nomenclature

$\begin{array}{ll}n_{i}, n_{e} & =\text { Specie number density } \\ \mathrm{N} & =\begin{array}{l}\text { Ambient gas number } \\ \text { density }\end{array} \\ v_{i}, v_{e} & =\text { Specie velocity } \\ \phi & =\text { Electrostatic potential } \\ \mathrm{E} & =\text { Electric field } \\ \mathrm{D} & =\text { Specie diffusivity } \\ \mathrm{M} & =\text { Specie mass }\end{array}$

$$
\begin{array}{ll}
\mathrm{e}, \mathrm{q}_{\mathrm{i}} & =\text { Specie charge } \\
\varepsilon_{0} & =\text { Permittivity of free space } \\
\mathrm{k} & =\text { Boltzmann constant } \\
S_{i e} & =\text { Specie collision rates } \\
\varepsilon_{k} & =\text { Internal energy density } \\
\mu & =\text { Specie mobility }
\end{array}
$$

\section{Introduction}

Recent work [1-12] on the efficient generation of ionized fluid in a glow discharge at atmospheric pressure using a dielectric barrier discharge (DBD) technique has attracted interest from the fluid dynamics and control communities. The largely athermal surface plasma generation investigated in the above studies can be used for achieving flow control by modifying the flow structure through electro-hydrodynamic

${ }^{1}$ Ph.D. student

${ }^{2}$ Adjunct Associate Scientist

${ }^{3}$ Clarence L. "Kelly" Johnson Collegiate Professor and Chair, Fellow AIAA 
(EHD) effects [1]. A typical flow control application is illustrated below where an insulator separates the electrodes powered by a radio frequency $(1-50 \mathrm{KHz})$ alternating voltage $(1-20 \mathrm{KV})$ for actuation at atmospheric pressure. The collisional plasma under such pressures can result in significant momentum exchange with the neutral species. For a given geometry the applied voltage and frequency generates a discharge with characteristics of a DBD, ionizing the neutral gas. A typical actuator configuration is shown in Figure 1, in an asymmetric arrangement with an exposed electrode and an insulated lower electrode. Figure 1 shows the effect of the plasma generated EHD flow on a surface as can be seen from the bending of the jet and re-emerging as a wall jet. This can be used to inject momentum in regions of adverse pressure gradients, boundary layers, and high heat flux. Compared to mechanical devices such as the synthetic jet [13], the glow discharge approach has the advantage of involving no moving parts. But before one can develop such a plasma actuator, more understanding of the physics is highly desirable in order to develop insight and tools for design and optimization of the device.

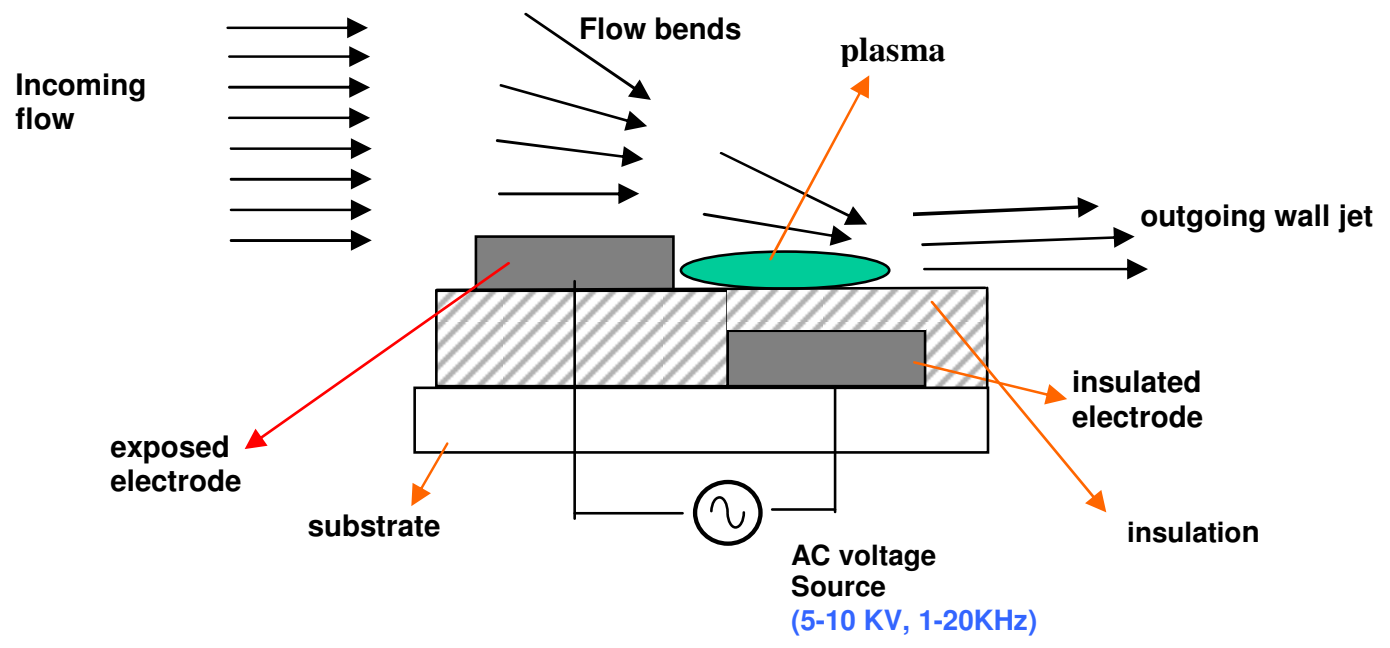

Figure 1 Illustration of Glow discharge Induced Fluid flow

The detailed mechanism of electrohydrodynamic (EHD) flow generation induced by the DBD is not clear although the concept behind the force generation is believed to be from collision-dominated effects. The paraelectric effects arising from the electric field gradient accelerate the ions which transfers momentum to the neutral fluid [2]. The effect of the plasma on the fluid can be modeled as a localized body force [1] on the neutral particles. Most of the investigations so far have been based on experimental observations and phenomenological arguments using simplified models [1,4]. The coupled plasma-fluid problem is inherently non-linear and exhibits wide ranges of time and length scales.

It is worth noting an important distinction here that the present EHD effects are more influential in low Reynolds number regimes, unlike the electromagnetic interaction of the ionized fluid in high Mach number conditions (say hypersonic regimes) where highly energized internal modes at temperatures around $5000 \mathrm{~K}$ are observed. The resulting ionization and the interaction with electromagnetic effects can alter flow conditions through Lorentz force. However, the velocity and the temperature coupling through Joule heating effects results in significant thermal effects. Such effects have been studied by, e.g., Rivir et al.[1415], Leonov et al.[16] and reviewed by Shang et al.[17].

\section{A. Experimental studies}

Experimental and analytical studies of atmospheric pressure glow discharge plasma-based flow were conducted by Roth et al.[2-4]. Here, EHD body force is modeled as an electrostatic force acting on the charged particles which acts on the neutral gas. Enloe et al.[5-7] studied the plasma morphology and operating mechanism using optical measurements using photomultiplier tube (PMT) which revealed the characteristic temporal and spatial structure. The studies revealed that discharge asymmetry and Debye 
shielding effects play a key role in the generation of EHD-induced flows. Shyy et al.[1] proposed that the asymmetries introduced through the electrode arrangement and the dielectrics along with the asymmetric discharge structure had a significant role to play in the generation of EHD-based paraelectric flow or simply glow-discharge-induced flow.

Parametric studies of a DBD actuator were conducted by Van Dyken et al.[10] where design parameters such as input signal waveforms, input signal frequency, geometric dimensions etc., were varied to study their effect. Most of the above studies addressed the observations through empirical arguments which mostly hypothesize possible physics. In trying to improve the operating design, Corke et al.[8] used a plasma array with phased inputs to investigate unsteady flow control. Separation control on a NACA66 wing was studied by Post et al.[11]. The plasma operation significantly reduces the size of the separation bubble. Chan [21] applied plasma-based EHD flow control in the study of acoustic effects in subsonic cavity flows.

There have also been efforts to use plasma-based actuators in the context of turbulent boundary layer flow control. EHD-based turbulent flow control concepts have been presented in recent articles by Soldati et al. [22-23]. The EHD flows affect the turbulence field by increasing both dissipation as well as production, thus maintaining the balance. However, overall drag reduction was observed with consistent decrease in the Reynolds stresses. The ionization in these cases is through electrostatic precipitators and efforts are being made to use the atmospheric pressure DBD in this context. Wilkinson [24] investigated the use of an oscillating surface plasma wave for turbulent drag reduction.

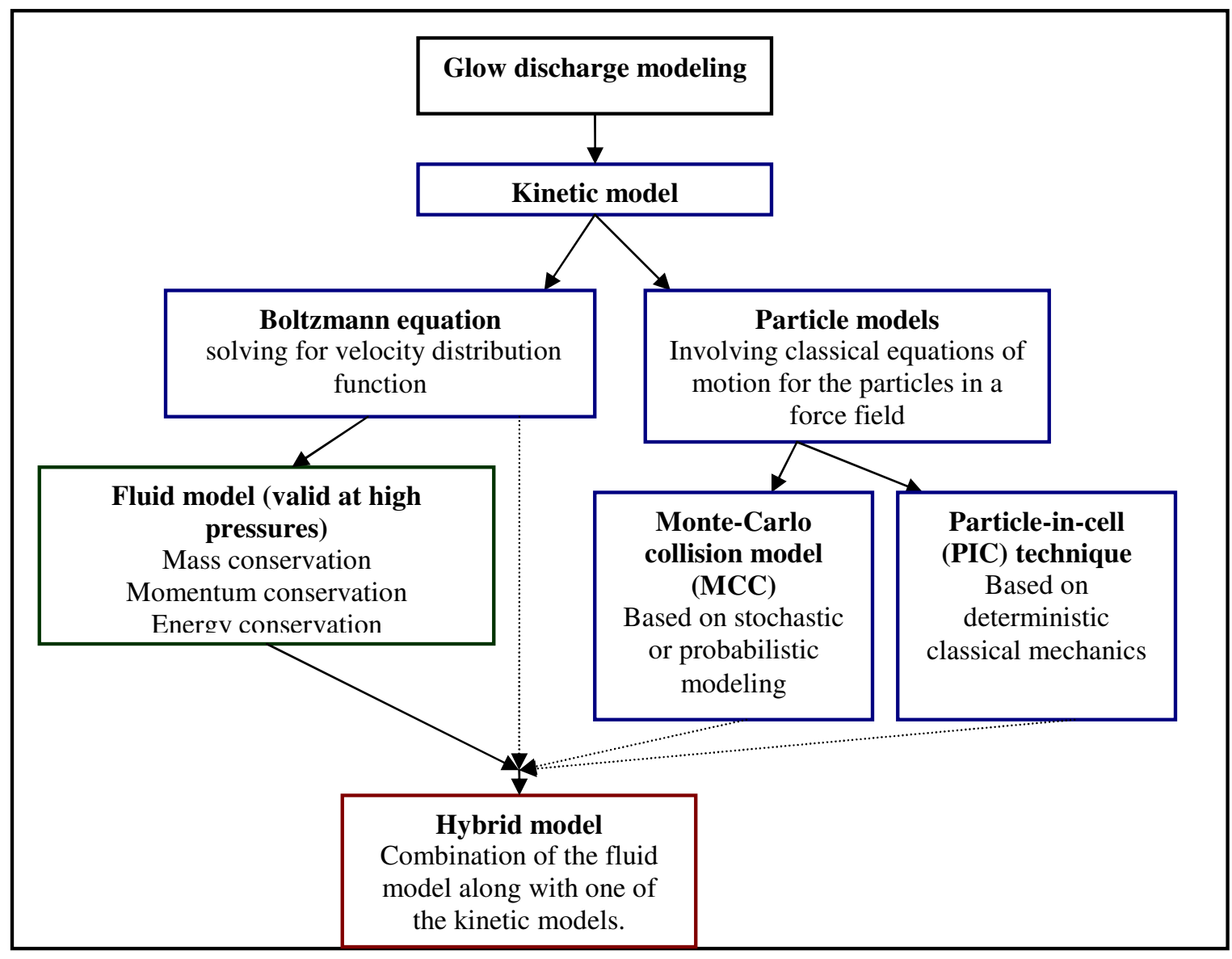

Figure 2 Plasma modeling hierarchy

\section{B. Numerical studies}

While experimental studies have been abundant, numerical efforts have been less forthcoming. Preliminary numerical studies employed analytical techniques in trying to simplify the plasma-fluid interaction such as those by Enloe et al.[5-7] and Roth et al.[2,3]. Our previous effort [1] focused on using 
an analytical-empirical model assuming a linear force distribution in the domain to approximate the discharge structure. The resulting flow field caused by the body force revealed qualitatively similar results to those observed experimentally although the model was simplistic.

Computational modeling of discharge plasmas can be achieved using a variety of approaches. The modeling complexity can be classified mainly into three types, namely, fluid models, kinetic/particle models and hybrid approaches, as shown in Figure 2. Kinetic models involve the solution of the Boltzmann equation [32] for the specie velocity or energy distribution function in both space and time or particle simulations, often using Monte Carlo methods and are generally computationally more expensive than the fluid models. The fluid models consist of a few moments of the Boltzmann equation. However, the choice of the model is also dependent on the regime of modeling interest. The coupling between the Boltzmann equation and the Poisson's equation for electrostatics is a difficult numerical problem. The particle dynamics should include the strong body force effect of electrostatics. Particle techniques such as MonteCarlo methods are used to model the collision term. Particle-in-cell (PIC) techniques have been used for discharge modeling in [27-29]. The PIC technique is based on deterministic classical mechanics of particles with prescribed force fields. Computational time is a key factor in such methods with the need to handle multiple scales and consequently time-averaged description is employed for faster processes. Hybrid approaches have also been tried for speed up, by, e.g., Riley et al.[32]. These methods are primarily used for low-pressure discharges where computational accuracy of the model is important along with efficiency. For example, Surendra et al.[35,36] have adopted such an approach where the slower particles are treated as a fluid while the kinetic models are employed for faster species far from equilibrium. However, for the simulation of higher pressure discharges $(\sim 100$ torr or higher), the velocity probability distribution function can be assumed to be close to equilibrium and, therefore, fluid models can adequately capture the relevant physics [27]. We will focus on the fluid models in the present study.

Fluid models have been used to describe both high- [37-42] and low-pressure [43-46] discharges. Colella et al. [45-46] and Hammond et al.[44] focused on the solution methodology of the discharge fluid equations. Colella et al. [45-46] employed a conservative finite difference method for low-pressure discharge modeling using local grid refinement in 2-D. High-pressure discharges were studied predominantly in 1-D situations by Massines et al., [37] etc. However, multi-dimensional studies [49-52] at atmospheric pressures have been attempted only recently, in the context of modeling DBD plasma-induced flow effects.

The key to modeling the DBD effects in fluid dynamics is to achieve realistic distributions of the species densities and their momentum in the domain which interacts with the neutral fluid by solving the plasma fluid equations. In our previous work, an analytical-empirical model [1] based on a linear force distribution in the domain served as a preliminary effort to model such phenomenon. This linearized body force model was later adopted by Gaitonde et al. [48] for modeling plasma-based separation control in a NACA 0015 wing section. This linearized body force model was an attractive option because of the difficulty in achieving efficient multi-dimensional and self-consistent plasma dynamics simulations coupled with the fluid dynamics. More recently, Roy et al. [49-50] proposed a self-consistent twodimensional DBD fluid model for helium gas with application to separation control using finite element techniques. This multi-fluid formulation to model the radio-frequency discharge in helium gas gives the spatial and temporal evolution of the charges species which is decoupled from the neutral fluid dynamics. The body force calculated from this data provides a more self-consistent way of modeling the plasma-wall jet interaction. Singh et al.[51], in a related paper, present a parametric study of the different conditions in an asymmetric discharge configuration. Specifically, it was observed that the net body force production in the domain over a whole time-cycle produced a downward positive force for a configuration as shown in Figure 3. Kumar et al.[52] study the nature of the discharge and the resulting force field in the presence of a magnetic field. Also studied is the shape effect of the electrodes in the event of a finite electrode thickness. All the above-mentioned modeling studies assume negligible electrode thickness. In the event of a finite-electrode thickness, the treatment of the dielectric-electrode edge can impact the near-wall force field. The common approach to be employed in the present study as well as in the previously mentioned modeling studies is that the plasma species and the neutral fluid are treated as a two-fluid system coupled through dynamic forces and pressure interactions.

The plasma fluid model has a wide range of operating length and time scales characterizing both the discharge and flow physics resulting in a highly stiff system of equations and is a major limiting factor in achieving a fully-coupled multi-dimensional plasma-fluid simulation. Similar stiffness is also observed in diverse problems such as modeling of chemically reacting flows [53], atmospheric chemistry [55] etc. 
These stiff systems typically restrict the capability of computational simulations through very small timestep size restrictions and hence warrant efficient solution procedures, especially during time-accurate studies. In this paper, we present our efforts towards clarifying some of the important computational issues pertaining to handling the multiple scales while offering insight into improving our computational capability for modeling practical, multi-dimensional physics. The results presented are part of an ongoing effort to study fully self-consistent plasma-dynamics in conjunction with the resulting fluid dynamics.

Regarding the multiple scales in the fluid model discharge equations, the solution procedure for such systems requires handling of various time scales corresponding to the different processes namely transport (convection and diffusion), reaction/ionization, dielectric relaxation, etc. efficiently. Both implicit and explicit techniques can be used to march in time with the choice usually based on the extent of stiffness of the system, expected time accuracy etc. Globally implicit integration methods are attractive in the sense that they are unconditionally stable, but are computationally burdensome in certain highly non-linear situations. This is necessitated by the need to find the root to a highly non-linear system which might entail very small time-steps to achieve convergence. Also, there is a burden of huge computational requirements. This method works reasonably well when the overall timescales are not very far apart.

Both sequential and fully implicit approaches have been used for discharge modeling. Roy et al.[4952] used a globally implicit finite element procedure where the system of specie continuity and momentum equations are assembled as part of a global matrix to solve for the solution vector. Sequential approaches have also been employed such as in the study of Hammond et al.[44] where a hybrid implicit-explicit formulation is used. The ions being slower are treated by an explicit $4^{\text {th }}$-order Runge-Kutta method followed by an implicit treatment of the fast electron equations. The implicit Euler (first-order) and implicit Runge-Kutta (second-order) methods were used with Newton-Raphson iterations to overcome the nonlinearity. Further, the resulting Jacobian was simplified by neglecting the weak off-diagonal contributions reducing to a block tri-diagonal system. However, the computational overhead from the iterative procedure for the fully implicit treatment and the cost of inverting the matrix more than compensates for the gain over explicit procedures. In another modeling study, Colella et al. [45-46] employed a time-split predictorcorrector procedure to enhance coupling, but still used a semi-implicit procedure for the integration of the individual equations. Another alternative to the implicit procedures used above is the use of matrix free Newton-Krylov methods, which have been reviewed in [60].

\section{Overview of present study}

In the present multiple scale problem, we have employed an operator-split sequential solution algorithm. Some examples of studies which employed such ideas are Najm et al. [53] in combustion problems, Verwer et al. [55] in atmospheric chemistry modeling and Tyson et al. [61] in chemotaxis models. Here, the solution procedure can be adapted to handle the individual processes efficiently and realize overall gain in computation. However, such an approach requires careful attention to stability considerations and performance is highly dependent on the physics of the problem. In certain situations the presence of competing stiff processes can lead to a system with much larger dynamical time-scale than dictated by the individual processes. Such dynamic equilibrium usually cannot be predetermined in general for a non-linear advection-diffusion-reaction system. The stiffness of the reaction part is typically overcome by using stiff integration procedures in ODE integration packages [56] such as the ones based on the backward-difference formulae (BDF). Alternative time-integration methods such as implicit-explicit Runge-Kutta methods [54] also exist. In using time-split algorithms for processes operating in a range of time-scales, the choice of time-step size is typically determined by the smallest time scale, but need not necessarily be chosen as such. To speed-up the solution procedure, an intermediate time scale is chosen to advance the overall system in time, while the faster processes are advanced by sub-cycling within the timestep. In the present study, the time-step dictated by the slower ion species convection is targeted to march the full discharge system while sub-cycling is used for the faster processes. Also, a predictor-corrector approach is employed to ensure sufficient coupling between the electric field and the specie densities. A strong coupling is essential for achieving stable time-accurate simulations while using a sufficiently large global time-step. This method is integrated with a multi-block finite-volume algorithm capable of handling 3-D curvilinear-grids [59]. The method is employed to model the plasma dynamics in an asymmetric electrode configuration similar to that shown in Fig. 1. We also investigate the effect on the flow field using the resulting body force field in a low Reynolds flow regime. The present study is part of an ongoing effort to develop a capability to study fully coupled multi-dimensional plasma-dynamics with the fluid flow. 
The rest of the paper is organized as follows. Section II presents the plasma fluid model along with the techniques employed to handle multi-scale discharge dynamics and the Navier-Stokes equations for fluid flow. This is followed by the computational setup in section III. In section IV, we present some representative results of 2-D helium discharge modeling efforts in an asymmetric electrode arrangement and the impact of the body force field on fluid flow. We conclude in section $\mathrm{V}$ and discuss future research directions.

\section{Numerical Model}

\section{A. Fluid model for discharge equations}

The fluid model used consists of the first few moments of the Boltzmann equation for the various species with a near-Maxwellian distribution function. However, as discussed previously, the validity of the fluid type description of the plasma is determined by the existence of a local thermodynamic equilibrium and conditions such as the operating pressure and the nature of the external force field. Nitschke et al. [27] compare the particle-in-cell model with a fluid model simulation of a radio frequency discharge in helium and conclude that considerable agreement between the studies was observed for pressures greater than 100 mTorr, which is much smaller than the atmospheric pressure regime of $\sim 760$ Torr. As a result, most of the high-pressure discharge simulations use fluid models which are potentially attractive for efficient computational studies if the limitations to their validity are adequately addressed. The number of species is typically dependent on the extent of plasma chemistry included in the model. The plasma is considered as a multi-component fluid comprised of two types of primary species, namely, ions and electrons. The conservation equations for specie density, momentum and energy equations are presented below.

\section{(a) Governing Equations}

The governing equations describing the discharge dynamics consist of the continuity, momentum and energy equations, respectively. For simplicity, we will consider just two species for the present discussion, namely, ions and electrons given by subscripts 'i' and 'e' respectively.

Continuity equation:

Electrons: $\frac{\partial n_{e}}{\partial t}+\nabla .\left(n_{e} v_{e}\right)=n_{e} S_{i e}-r n_{i} n_{e}$

Ions: $\quad \frac{\partial n_{i}}{\partial t}+\nabla \cdot\left(n_{i} v_{i}\right)=n_{e} S_{i e}-r n_{i} n_{e}$

Here, the source terms on the right hand side represent the reaction/ionization processes which result in the creation $(\mathrm{S})$ or destruction $(\mathrm{r})$ of the species, as applicable. It is very common to write the ionization coefficient $\mathrm{S}$ as a function of $\mathrm{E} / \mathrm{N}$.

\section{Momentum equation}

The momentum equation for the species at high pressures can be reduced to the drift diffusion form which neglects the inertial and unsteady terms and balances the thermodynamic pressure gradient with the drift force and collision terms.

Electrons: $n_{e} \mu_{e} E-\nabla\left(n_{e} D_{e}\right)=n_{e} v_{e}$

The drift-diffusion approximation is a common choice for high-pressure discharges while the full momentum equation is required for low-pressure situations.

Ions: $\quad n_{i} \mu_{i} E-\nabla\left(n_{i} D_{i}\right)=n_{i} v_{i} \quad$ (for high-pressure discharges)

Such an approximation requires the pressure tensor to be isotropic and is strictly valid only for a Maxwellian distribution. As long as the thermal velocity is comparable to the drift velocity and we are in the continuum regime $\left(\frac{\lambda}{L}=K n \ll 1\right)$, the inertial components in the momentum equation can be neglected. What is realized is a balance between the collision/ionization effects and the drift-diffusion components. By using a similar scaling argument, the energy equation can also be assumed to achieve local thermodynamic equilibrium instantaneously due to the high pressures. This renders the local energy density a function of the local reduced electric field $(\mathrm{E} / \mathrm{N})$. The specie energetics is usually linked to the continuity and momentum equations through the ionization/reaction coefficients, diffusivity, mobility, etc. which is 
usually modeled based on the local energy density (or in this case E/N). However, this cannot be assumed if Joule heating and other thermal effects are significant and need to be modeled.

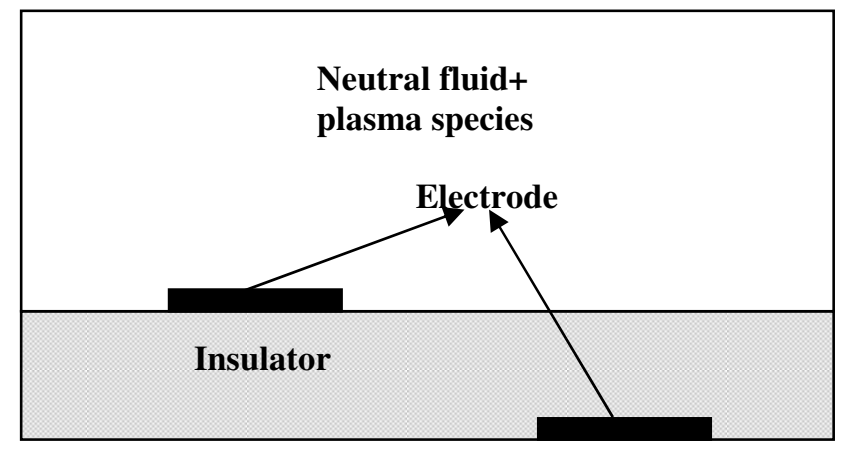

(a) 2-D actuator arrangement

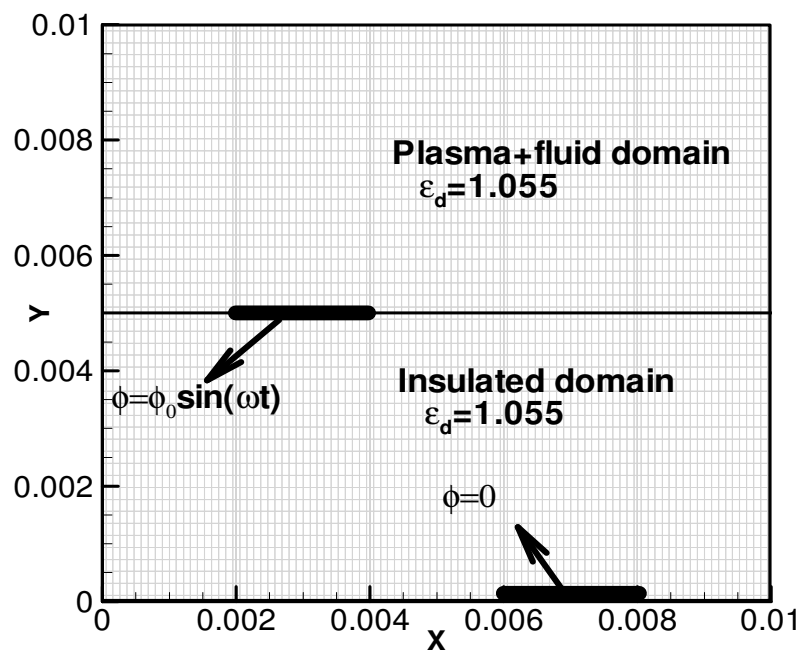

(b) 2-D Computational domain with grid

Figure 3 A representative 2-D asymmetric discharge arrangement

\section{Electric field equation}

The electric field $\mathrm{E}$ is obtained using the solution of the Poisson equation, given by

$\nabla .\left(\varepsilon_{d} E\right)=\frac{e\left(n_{i}-n_{e}\right)}{\varepsilon_{0}}$

Defining a source/ionization frequency to simplify the source term, we can write $v_{c}=S_{i e}-r n_{i}$

We denote the reference number density, specie velocity, length, time, electric field by $\mathrm{N}, \mathrm{V}_{\mathrm{i} / \mathrm{e}}, \mathrm{L}, \tau$ and $\mathrm{E}$, respectively. To give an idea of the characteristic time-scales in the system, their representative orders of magnitude based on an RF discharge operating at a few KHz based on helium gas [47] are given in Table 1.

\section{(b) Solution algorithm}

In the present study we propose an operator-split algorithm which is capable of efficiently handling the different time scales arising from the various processes such as ionization, convection, diffusion etc. The flexibility to use a variety of choice of methods for the different operators presents itself 
to both implicit and explicit handling as is warranted. This time-split integration algorithm is embedded as part of a projection method to enhance coupling between the various specie equations being solved. In the following section, an outline of the algorithm is presented.

The split solution algorithm consists of the following steps:

1. Predictor step

2. Solving the Poisson equation

3. Corrector step

Table 1 Representative Time Scales in the problem

\begin{tabular}{|c|c|c|}
\hline \multicolumn{2}{|l|}{ Time scale } & \multirow{2}{*}{$\begin{array}{c}\text { Order } \\
T\end{array}$} \\
\hline Operating frequency timescale, $\tau_{\omega}$ & $\tau=\tau_{w}=\frac{1}{f}=T$ & \\
\hline Ion drift $\tau_{d r, i o n}$ & $\tau_{d r, i o n}=\frac{L}{V_{i o n}}$ & $\mathrm{O}\left(10^{-4}\right) T$ \\
\hline Electron drift $\tau_{d r, e}$ & $\tau_{d r, e}=\frac{L}{V_{e}}$ & $\mathrm{O}\left(10^{-6}\right) T$ \\
\hline Dielectric relaxation $\tau_{\text {diel }}$ & $\tau_{\text {diel }}=\frac{\varepsilon_{0} E}{e N V_{e}}$ & $\mathrm{O}\left(10^{-7}\right) T$ \\
\hline Ionization timescale $\tau_{c}$ & $\tau_{c}=\frac{1}{v_{c}}$ & $\mathrm{O}\left(10^{-6}\right) T$ \\
\hline
\end{tabular}

1. Predictor step :

At the beginning of the $\mathrm{n}+1^{\text {th }}$ time level and for the $\mathrm{k}^{\text {th }}$ specie, we have $n_{k}^{n}, v_{k}^{n}, E^{n}$ and $v_{k}^{n}$ as the known quantities. Here the specie continuity equations (1)-(2), along with the drift-diffusion momentum equations (3)-(4) are integrated using lagged values for the various coefficients (as they are a function of the electric field E). The source term is integrated using a higher-order ( $4^{\text {th }}$-order) BDF using the CVODE solver [63]. The convection and diffusion operators can be treated either implicitly or explicitly. In this case, we will employ a second-order upwind for the convection term and second-order central difference for the diffusion term. The continuity equation can be written as

$\frac{\partial n_{k}}{\partial t}+\nabla \cdot\left(n_{k} v_{k}\right)=v_{c, k} n_{k}$

As can be seen from Table 1, the presence of processes of disparate time scales can possibly be better handled using operator splitting. Three types of splitting are popular, namely the standard first-order splitting, Strang splitting and source splitting.

(i)first-order splitting

The first-order splitting can be written symbolically as

$\bar{n}_{k}=T^{M_{k}}\left(\Delta t^{\prime}\right) S(\Delta t) n_{k}^{n}$

where $\mathrm{S}$ is the reaction operator integrated using the ODE solver CVODE [63] and $\mathrm{T}$ is the transport operator. $M_{k}$ is the number of substeps used for the transport term integration to march to the global timestep $\Delta t$. Therefore we have

$\Delta t^{\prime}=\frac{\Delta t}{M_{k}}$

\section{(ii) Strang splitting}

Here, the transport term integration is usually split into two halves to achieve the symmetry since the ODE solver used in the reaction part is more computationally burdensome. 
$\bar{n}_{k}=T^{M_{k} / 2}\left(\Delta t^{\prime}\right) S(\Delta t) T^{M_{k} / 2}\left(\Delta t^{\prime}\right) n_{k}^{n}$

(iii) Source splitting

In this study, we limit the discussion to first-order source splitting [54]. The source splitting has certain advantages over the standard first-order splitting and the higher-order Strang splitting methods. The Strang splitting is formally second-order which is achieved by splitting the operators symmetrically. It is worth noting that, in both the first-order and Strang splitting procedures, the initial guess for the reaction part is not directly from the previous time-step, but after a half or full time-step of the transport term integration. This results in the introduction of stiff transients in the solution which are nothing but an artifact of the splitting errors. These can be highly significant in the presence of strong non-linearities. Even though Strang splitting is formally second-order, this is rarely achieved [62] for certain stiff problems where it is known to deteriorate to a lower order in the presence of heavy stiffness. To overcome the solution discontinuities which gave rise to stiff transients in the above two splitting methods, the source splitting treats the transport as a piecewise constant source. For example, we can write

$\tilde{n}_{k}=S(\Delta t) n_{k}^{n}$

and

$\dot{n}_{k}=\frac{\tilde{n}_{k}-n_{k}^{n}}{\Delta t}$

Therefore, we have

$\bar{n}_{k}=\left(T\left(\Delta t^{\prime}\right)+\dot{n}_{k}\left(\Delta t^{\prime}\right)\right) n_{k}^{n}$

where $\mathrm{S}$ is the reaction operator integrated using the ODE solver CVODE [63] and $\mathrm{T}$ is the transport operator. $M_{k}$ is the number of substeps used for the transport term integration to march to the global timestep $\Delta t$. Thus, we have

$\Delta t^{\prime}=\frac{\Delta t}{M_{k}}$

The ODE solver employs the following elements

1) a $5^{\text {th }}$-order BDF for time integration

2) Newton iteration for non-linearity

3) a direct method with a banded treatment of the Jacobian

4) normal mode with subcycling within the time-step

5) relative and absolute tolerances of $1 \mathrm{e}-12$ and 1e-14 respectively.

The above strict tolerances were chosen so that the ODE integration is almost exact.

2. Solving the Poisson equation

Now that we have the predicted values of the specie densities, $\bar{n}_{k}$ we can use it to update the electric field by solving the following Poisson equation:

$\nabla .\left(\varepsilon_{d} E^{n+1}\right)=\frac{\sum_{k} \bar{n}_{k} q_{k}}{\varepsilon_{o}}$

where $q_{k}$ is the signed charge content of each specie. The elliptic solver employed in the present study is either the Gauss Seidel method with successive over-relaxation or the algebraic multigrid method.

\section{Corrector step}

At the end of the previous step, the predicted specie densities $\bar{n}_{k}$ and the electric field at the new time level $\mathrm{E}^{\mathrm{n}+1}$ are available. Now we will obtain the corrected densities at the new time level. It is worth noting here that the corrector step is needed to ensure adequate coupling between the electric field and the specie number density especially for the convection term. In other words, we require appropriate coupling between the specie continuity equation and the momentum equation (or the drift-diffusion equation). Hence, the corrector step is the same as the predictor step, but performed with the updated coefficients using $E^{n+1}$. It is also worth noting that the if the diffusion term and the source terms are not strongly 
impacted by the electric field, then the corresponding operators can be removed from the corrector step as correction is needed to be done only for the convection term in such cases.

\section{(c) Accuracy, stability and efficiency}

One of the important aspects is to determine the proper choice of the global time-step $\Delta t$ and the number of sub-steps $M_{k}$ which would require balancing computing efficiency with stability. Choosing $\Delta t$ too small will make the computation highly inefficient and a reasonable choice in this case is the slower specie convection time-scale. Also, issues such as dynamic equilibrium and non-linearity can affect the solution procedure and the size of the global time-step. While sub-cycling is an important technique for improving accuracy and stability, its ability to increase the global time-step is limited. It is important to gauge the overall accuracy with the global time-step size.

The criterion for stability of the above predictor-corrector approach with stiff sub-steps is not straightforward. Of the time scales listed in Table 1, the ionization source term and the dielectric relaxation time step restrictions are not binding in the above algorithm. The semi-implicit treatment for the reaction source terms makes it unconditionally stable. The predictor-corrector formulation with the solution of the Poisson equation ensures that the space charge stability restriction is slightly relaxed. With all the split integration procedures being explicit for the convection, the stability of the system will be determined by the CFL conditions for the individual steps. The global integration time-step, $\Delta t$, is determined by the slowest time scale. Here, $\lambda$ is the CFL number for the different species, given by

$$
\lambda_{k, C F L}=\left|v_{k}^{\max }\right| \frac{\Delta t}{\Delta x}
$$

In the case of a number of different species considered, the choice of the global time-step is usually the convection time scale of the slowest specie, unless there is a large range of variation. This is important if we are considering the neutral species in reaction chemistry whose dynamics is determined by the slower moving neutral fluid. However, this might not always guarantee a stable solution since the system is nonlinear. Hence, a stability range needs to be identified for the overall system of equations. Once this is fixed, the time-step for the faster electron species and the corresponding number of sub-steps M, can be obtained, so as to satisfy the electron CFL limit. However, studies [53] indicate that the CFL stability limit of the split algorithm deviates from that of unsplit algorithm depending on the number of sub-steps, $\mathrm{M}$ employed. Knio et al.[54] show that the critical CFL number due to transport operator sub-stepping decreases monotonically as the number of sub-steps increases in two-dimensional modeling studies of reacting flows. Also, one-dimensional studies indicate a limiting value of the critical CFL being achieved as the number of sub-steps is increased. In spite of the stability criterion becoming stringent, the overall computational savings can be substantial.

\section{B. Navier-Stokes solver}

The neutral fluid is modeled using the Navier-Stokes equations and the energy transport equation for a steady, incompressible flow. The body force terms, which are added to the momentum equations, couple the discharge effects to the fluid flow. The fluid is assumed to be incompressible in view of the plasma being essentially isothermal. In the following, governing equations in the two-dimensional form are presented.

$$
\begin{aligned}
& \frac{\partial \bar{A}}{\partial t}+\frac{\partial \bar{B}}{\partial x}+\frac{\partial \bar{C}}{\partial y}=\bar{D}, \\
& \bar{A}=\left[\begin{array}{l}
\rho \\
\rho u \\
\rho v \\
e
\end{array}\right], \\
& \bar{B}=\left[\begin{array}{l}
\rho u \\
\rho u^{2}+p-\tau_{x x} \\
\rho u v-\tau_{x y} \\
u(e+p)-\tau_{x x} u-\tau_{x y} v-k \partial_{x} T
\end{array}\right]
\end{aligned}
$$




$$
\begin{aligned}
\bar{C} & =\left[\begin{array}{l}
\rho v \\
\rho u v-\tau_{x y} \\
\rho v^{2}-\tau_{y y}+p \\
u(e+p)-\tau_{x y} u-\tau_{y y} v-k \partial_{y} T
\end{array}\right] \\
\bar{D} & =\left[\begin{array}{l}
0 \\
F_{x} \\
F_{y} \\
0
\end{array}\right]
\end{aligned}
$$

The $\mathrm{F}_{\mathrm{x}}$ and $\mathrm{F}_{\mathrm{y}}$ are the body force terms calculated from the solution of the plasma dynamics, as

$$
F_{x}=E_{x} \sum_{k} q_{k} n_{k}
$$

and

$$
F_{y}=E_{y} \sum_{k} q_{k} n_{k}
$$

The Navier-Stokes equations are solved using a pressure based algorithm with a second-order upwind scheme as discussed in [57-58].

\section{Computational setup}

In this section, we will model the two-dimensional radio frequency dielectric barrier discharge (DBD) in helium gas at high pressure in an asymmetric configuration as shown in Figure 3. A similar setup has been employed by Roy et al. [49]. We will present results from this case using the numerical method discussed above.

\section{A. Problem description}

A schematic of the computational domain is shown in Figure 3.The electrodes are $2 \mathrm{~mm}$ in length and the insulated bottom electrode is shifted downstream by $2 \mathrm{~mm}$. A similar case has been studied by Roy et al.[49]. The helium discharge is modeled at a pressure of 300 Torr, and a temperature of $300 \mathrm{~K}$ and is driven by an $\mathrm{AC}$ voltage of $1.5 \mathrm{KV}$ (peak voltage) operating at $5 \mathrm{KHz}$. The thickness of the electrodes is negligible and the dielectric thickness is $5 \mathrm{~mm}$. The electron temperature is assumed to be $1 \mathrm{eV}(\sim 11600 \mathrm{~K})$, while the ions and the neutrals are essentially in thermal equilibrium at $300 \mathrm{~K}$. The various transport properties and property relationships are available in literature and we will use the ones used by Roy et al.[49] for the present study. These are summarized in Table 2.

The computational domain employed is a square domain of size $1 \mathrm{~cm} \mathrm{x} 1 \mathrm{~cm}$. The grid consists of $127 \times 61$ points for the 2-D case as is shown in Figure 3b. The dielectric constant in the fluid/discharge domain is 1.00555; the permittivity of vacuum and that of the insulator is 4.5 . For investigation purposes two different frequencies are considered, namely, $5 \mathrm{KHz}$ and $10 \mathrm{KHz}$, respectively, with all other conditions remaining constant. A global time-step of $10^{-8} \mathrm{~s}$ is used for the computations. The initial number density in the plasma is $1.0 \times 10^{15} / \mathrm{m}^{3}$ for all the different species.

\section{B. Boundary conditions}

The homogeneous Neumann boundary condition is applied for the electrostatic potential at the open boundaries while the Dirichlet boundary condition is used at the electrode:

At the exposed electrode: $\phi=\phi_{0} \sin (2 \pi f t), \phi_{0}=1.5 \mathrm{KV}$.

At the submerged electrode: $\phi=0$

For the plasma species modeling, the domain boundary away from the insulator/electrode surface is assigned a zero gradient condition assuming insignificant impact far away from the fluid-actuator interface. At the dielectric surface, the normal species flux is enforced to be zero. At the electrode, the treatment is slightly different. The electrons are assumed to be isothermal (at $11600 \mathrm{~K} \mathrm{or} 1 \mathrm{eV}$ ) at boundaries. At the exposed electrode, the thermal flux towards the wall is considered while it is neglected 
when the drift is away from the wall. Also, a weak secondary emission coefficient of 0.02 is used at the electrode. For the ions or the heavier species, the drift effects are significant and hence a zero gradient condition $\left(\frac{\partial n_{i}}{\partial n}=0\right)$ is applied.

For the neutral fluid, the insulator/electrode interface is assigned a no-slip condition for the momentum equation. A small mass flux is specified at the left edge of the domain to simulate a low Reynolds number flow, while at the other open boundaries, a zero velocity gradient condition is imposed considering negligible impact away from the wall. The Reynolds number is chosen to be small so that the effect of the discharge-induced flow field can be sufficiently observed.

Table 2 Summary of property models employed for the He discharge simulation [49]

\begin{tabular}{|c|c|}
\hline $\begin{array}{c}\text { Transport/reaction } \\
\text { properties }\end{array}$ & Models/values employed \\
\hline$\mu_{i}$ ( ion mobility) & $=\frac{8 \times 10^{3}}{p}\left(1-8 \times 10^{-3} E / p\right) \mathrm{cm}^{2} V^{-1} \mathrm{~s}^{-1}$ for $E / p \leq 25 \mathrm{Vcm}^{-1}$ torr $^{-1}$ \\
& $=\frac{4.1 \times 10^{4}}{p \sqrt{E / p}}\left(1-\frac{27.44}{(E / p)^{1.5}}\right) \mathrm{cm}^{2} V^{-1} \mathrm{~s}^{-1}$ for $E / p>25 \mathrm{Vcm}^{-1}$ torr $^{-1}$ \\
\hline$\mu_{e}$ (electron mobility) & $=\frac{e}{m_{e} v_{e n}} \mathrm{~cm}^{2} V^{-1} \mathrm{~s}^{-1}$ where $v_{e n}=10^{12} / \mathrm{s}$ \\
\hline$S_{i e}$ (specie ionization model) & $=4.4 \exp \left(\frac{-14}{(E / p)^{0.4}}\right) p \mu_{e} E \mathrm{~s}^{-1}$ \\
\hline$r$ (recombination coefficient) & $=1.09 \times 10^{-20} T^{-9 / 2} n_{e} \quad \mathrm{~m}^{3} / \mathrm{s}$ \\
\hline$D_{i}$ (ion diffusivity) & $=500 \mathrm{~cm}^{2} / \mathrm{s}$ \\
\hline$D_{e}$ (electron diffusivity) & $=\frac{k T_{e}}{e} \mu_{e} \mathrm{~cm}^{2} / \mathrm{s}$ \\
\hline$\mu$ (viscosity of He gas) & $=2.0 \times 10^{-5} \mathrm{Ns} / \mathrm{m}^{2}$ \\
\hline
\end{tabular}

\section{Results and discussion}

Using the above described computational capability, 2-D asymmetric dielectric barrier discharge in helium is modeled and the results are analyzed. In the following, the base set of results are presented for a frequency of $5 \mathrm{KHz}$ and a peak voltage of $1.5 \mathrm{KV}$ (peak voltage). To gauge the sensitivity of the solution with respect to the applied frequency, simulations at $10 \mathrm{KHz}$ were also studied.

In Figure 4, the axial electric field contours at two instants in time $\left(\omega t=\frac{\pi}{5}, \frac{\pi}{2}\right)$ during the positive half cycle are compared with the results of Roy et al.[49]. There is a reasonable agreement between the two results, although the present computations show slightly higher field strength near the electrode edges as compared to their results. The electric field in the positive half cycle where the upper electrode is positively charged is characterized by a strong positive electric field in the region near the inner edge of the two electrodes and strong negative field near the outer edges. The electrode-dielectric edge represents a discontinuity in the potential and hence the field strength on the edges is expected to be strong. The treatment of this discontinuity and consequently the calculation of the gradient (electric field) can significantly impact the overall solution and thus exact numerical comparison is difficult at this point. It has also been reported [52] that this jump in potential can affect numerical stability which has been overcome using artificial stabilization techniques by adding unphysical diffusion. However, no such treatment has 
been employed in the present study. A more extensive discussion on this issue is presented in [52]. Further investigation on this issue is required.

The region of strong electric field will be the region of extensive ionization in domain. However, the overall charge distribution is determined by the dynamics caused by the drift because of the electric field. The region of extensive charge accumulation is usually near the electrodes and the dielectric surfaces where the field lines are supposed to originate and end accordingly. In Figure 5, the distribution of the net charged specie number density (i.e $\mathrm{n}_{\mathrm{i}}-\mathrm{n}_{\mathrm{e}}$ ) is presented at $\omega t=\frac{\pi}{2}$. It is apparent that the bulk of the positive net charge is accumulated near the dielectric surfaces which act as the negative electrode for the positive half cycle. The force distribution (Eq. (22)-(23)) in the domain is a product of the net charge and the local field strength.

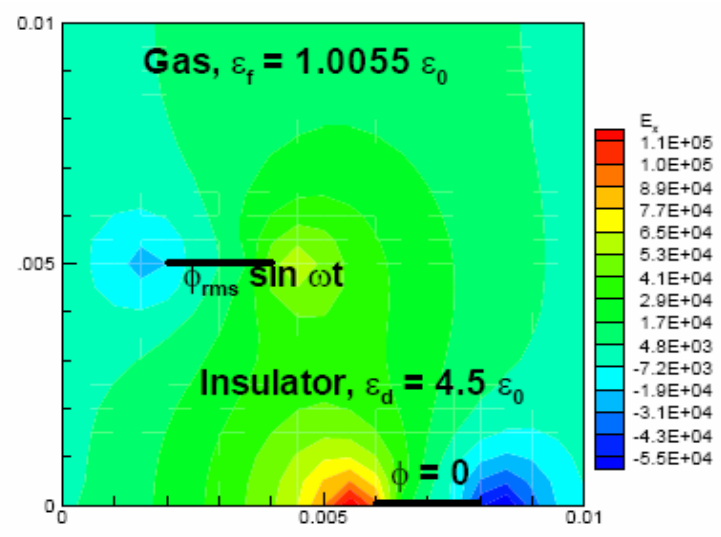

(a) $\omega \mathrm{t}=\pi / 5 \mathrm{rad}$

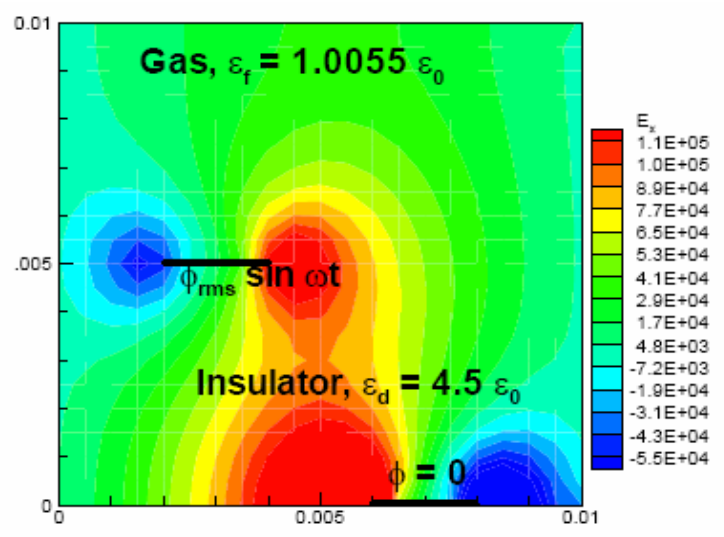

(b) $\omega \mathrm{t}=\pi / 2 \mathrm{rad}$

(a) Computations by Roy et al. [49](pictures have been reproduced form [49]).
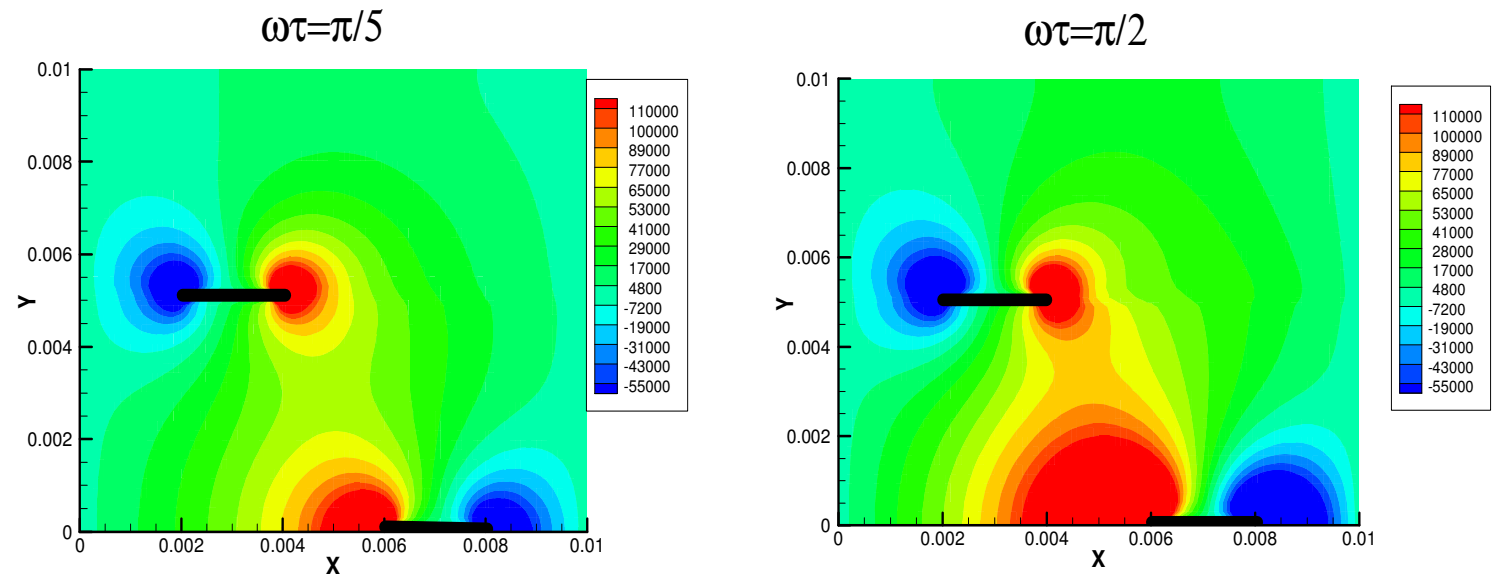

(b) Present computations

Figure 4 Axial Electric field (V/m) comparison for a $5 \mathrm{KHz}$ case between the present computations and that of Roy et al.[49] at two instants of the cycle at two instants of time (i) $\omega t=\frac{\pi}{5}$ (ii) $\omega t=\frac{\pi}{2}$ 
$\omega \tau=\pi / 2$

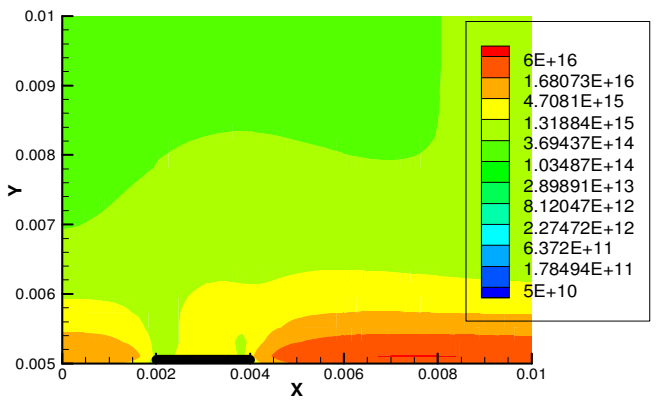

(a) $\omega \tau=\pi / 2$

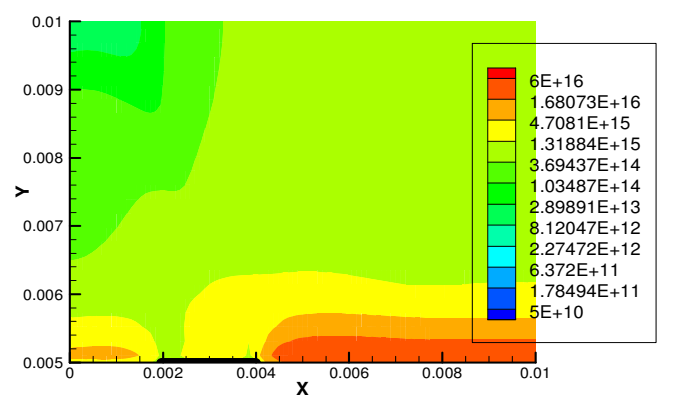

(b)

Figure 5 Contours of $n_{i}-n_{e}\left(\mathbf{m}^{-3}\right)$ for the two different frequencies (a) $5 \mathrm{KHz}$ (b) $10 \mathrm{KHz}$ at $\omega t=\frac{\pi}{2}$.

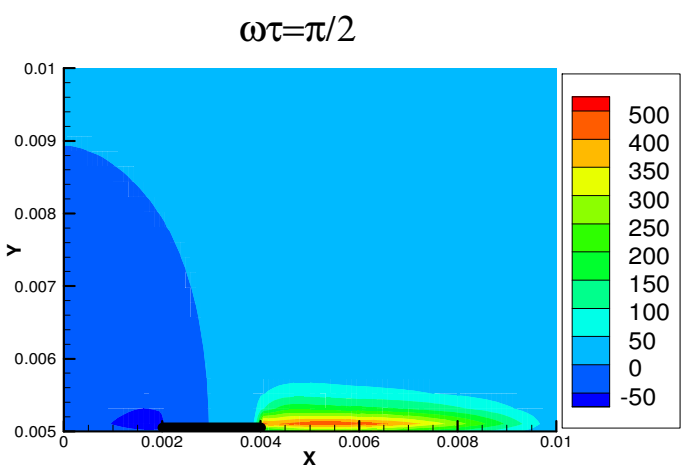

(a) $5 \mathrm{Khz}$ $\omega \tau=\pi / 2$

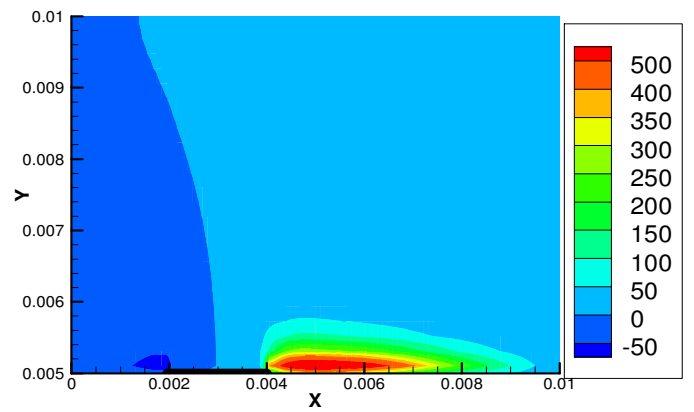

(b) $10 \mathrm{Khz}$

Figure 6 Contours of axial force density $\left(\mathrm{N} / \mathrm{m}^{3}\right)$ for two different frequencies (a) $5 \mathrm{KHz}$ (b) $10 \mathrm{KHz}$ at $\omega t=\frac{\pi}{2}$.

$\omega \tau=\pi / 2$

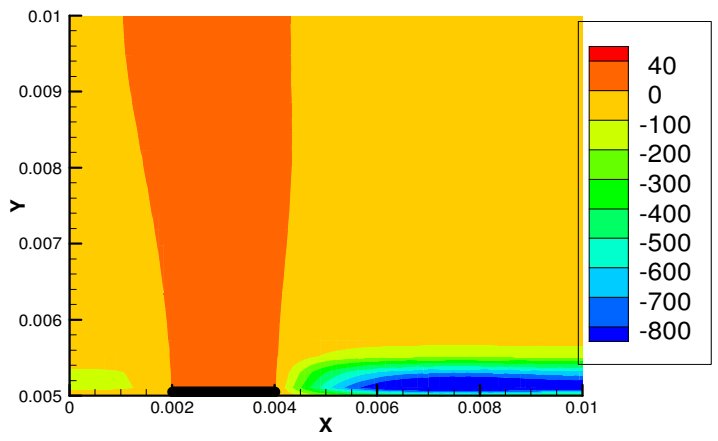

(a) $\omega \tau=\pi / 2$

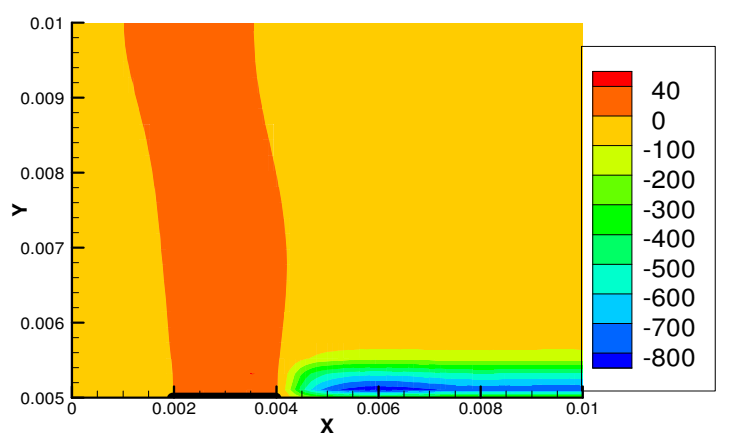

(b)

Figure 7 Contours of vertical force density $\left(\mathrm{Nm}^{-3}\right)$ for two different frequencies (a) $5 \mathrm{KHz}$ (b) $10 \mathrm{KHz}$ at $\omega t=\frac{\pi}{2}$. 
Based on the field strength and the charge distribution obtained above, the horizontal and the vertical force components are calculated. The force density contours are shown in Figure 6 and Figure 7 for two different frequencies at $5 \mathrm{KHz}$ and $10 \mathrm{KHz}$ at the instant $\omega t=\pi / 2$. The horizontal force for the $10 \mathrm{KHz}$ frequency shows a much larger force lobe and a stronger core as compared to the $5 \mathrm{KHz}$ case. This is to be expected since the driving voltage fluctuations are faster in time and hence, there is a much stronger momentum transfer effect. As for the vertical force components, they are predominantly negative in the whole domain except for the region above the electrode. This is caused by the vertical electric field components being upwards for the peak instant of the positive half cycle. However, the bulk of the charge accumulation being near the insulator surface renders this positive vertical force region rather weak. It is worth mentioning here, that the above factors were not adequately considered in the development of our linearized body force model, previously studied [1]. Specifically, the linear body force model differs from the above presented force model in the following manner:

(i) The linear force model assumes a strictly negative vertical force as against that obtained in the present study.

(ii) The linear force model assumes a smaller and thicker discharge region, while the present model predicts a longer and slender region.

(iii) The linear force model does not consider the plasma formation towards the left edge of the electrode.

While, the linear approximation of the force field is not necessarily bad in itself, the identification of the various empirical modeling parameters makes it less meaningful. It has been mentioned in empirical arguments [1] and in the interpretation of the experimental results [5-7] that asymmetry of the discharge geometry plays a significant role in the unidirectional flow generation. In order to verify this, the horizontal force field at two time instants in the positive and negative half cycle was studied for the $5 \mathrm{KHz}$ case.

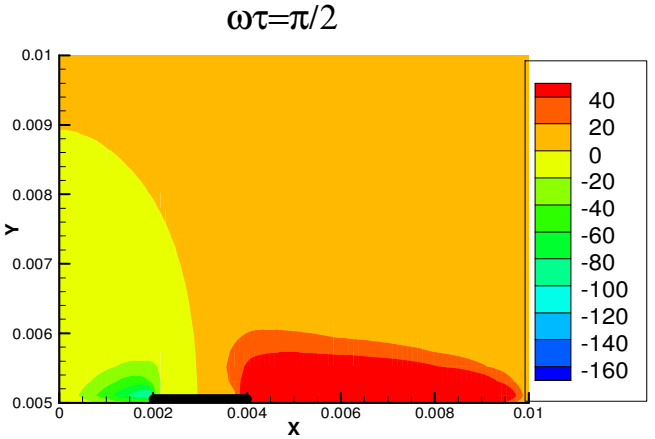

(a)

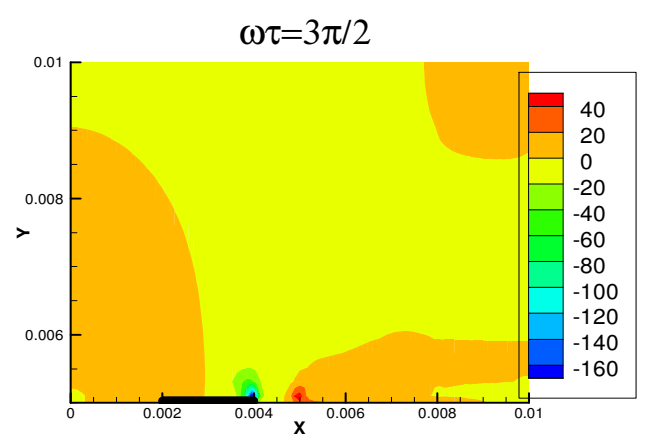

(b)

Figure 8 Horizontal force field $\left(\mathrm{N} / \mathrm{m}^{3}\right)$ contours for the $5 \mathrm{KHz}$ case at two instants of time (a) $\omega t=\frac{\pi}{2}$ and (b) $\omega t=\frac{3 \pi}{2}$.

In Figure 8, the horizontal force field at the peak instant of the positive and negative cycles is compared. It is apparent that during the positive cycle, the bulk of the domain has a positive force. During the negative half, the force is still positive in the region of wall jet formation, while the bulk of the domain is acted on by a weakly negative force. This disparity in the strength of the forces between the two half cycles when averaged over time causes a unidirectional flow effect. Now that the force field has been obtained, we can solve for the fluid flow by representing the plasma as a body force effect in the NavierStokes equations. In order to investigate this, we consider a very low Reynolds number flow of helium gas in the domain such that the plasma induced flow is pronounced. The computed force field obtained for a frequency of $5 \mathrm{KHz}$ at the instant $\omega t=\pi / 2$ is used as a body force in the fluid flow momentum equations. The results from the linear force model are also obtained for the same flow conditions for a comparison study. Figure 9 shows the velocity profiles at the two stations indicated on the adjacent velocity contour plot. The contour plots shows significant difference in the flow fields obtained for the two force 
distributions. The linear force model being of finite discharge size and of uniform net charge density shows a concentrated effect very near to the electrode. Also, force being present only in a small region from the electrode fails to suppress the diffusion effect and causes the streamlines to depart from the wall surface. The computed force field on the other hand, displays a much more uniform effect along the surface. The large discharge area as seen from the number density and the elongated nature of the horizontal force keeps the wall jet relatively close to the surface. The near wall velocity profiles are slightly weaker, but much flatter for the computed force field. The linear force model shows a slightly detached, but stronger wall jet. However, a more rigorous evaluation of the two models needs to be done using appropriate experimental results.
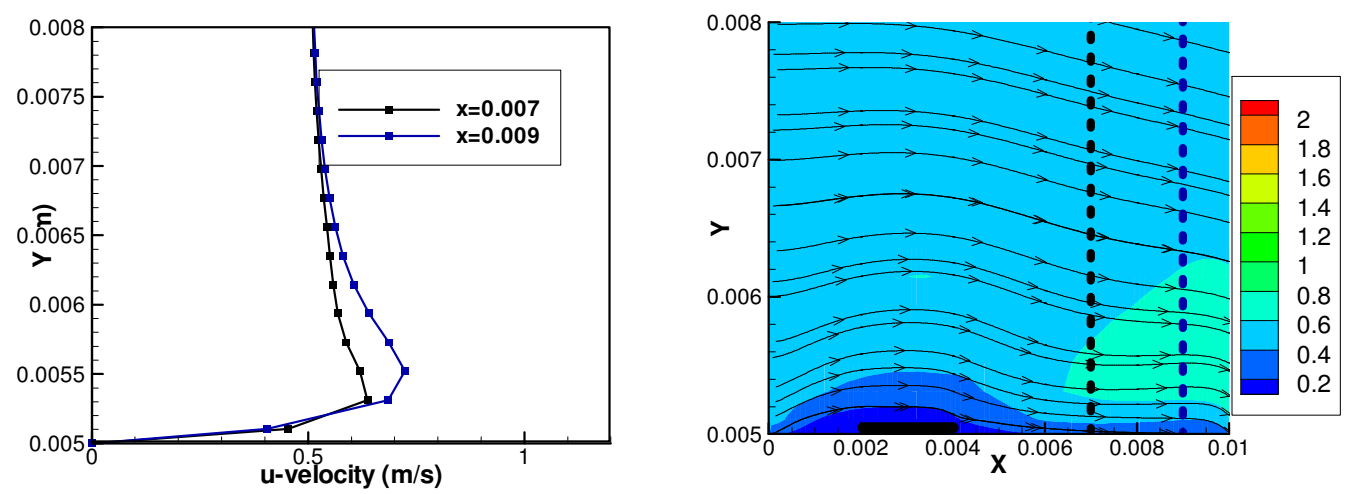

(a) Flow field and velocity profile using computed force field at $\omega \mathrm{t}=\pi / 2$
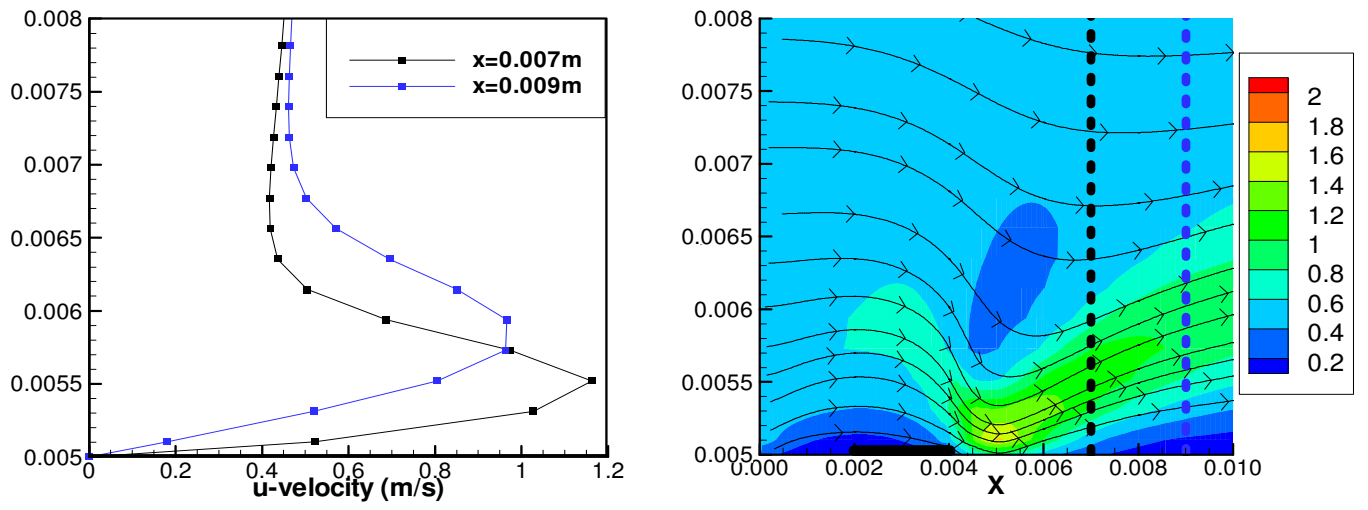

(b) Flow field and velocity profile using linearized force field

Figure 9 Comparison of the linearized and computed force fields (u-velocity contours) for a $5 \mathrm{Khz}$ case using the u-velocity contours $(\mathrm{m} / \mathrm{s})$. The velocity profiles are shown after subtracting the mean flow component.

\section{Conclusions and Future work}

A hydrodynamics model-based solution method for modeling the plasma dynamics in the context of a discharge-induced fluid flow is presented. This provides a way of self-consistent modeling of the discharge effects as opposed to an analytical-empirical model previously presented [1]. The twodimensional helium dielectric barrier discharge in an asymmetric geometry has been modeled using a finite volume operator-split sequential approach to efficiently solve the multi-scale problem. The time-dependent results obtained from the plasma equation were used to calculate the body force. The resulting body force distribution, while displaying overall qualitative agreement with the physics and observed effects, displays considerable deviation in performance from the simplistic linear force distribution assumption used before [1]. Specifically, the overall body force acts towards the wall and to the right of the exposed electrode for the present discharge configuration. The force comparison at similar time-instants in the two half-cycles exhibited different behavior, hence generating a uni-directional wall-jet type fluid flow. While overpredicting the velocity field, the linear force model also shows enhanced departure of the wall jet from the dielectric surface. Future work will involve addressing issues related to different types of splitting and the 
numerical accuracy of the solution algorithm. Furthermore, a fully coupled plasma dynamics-Navier Stokes solution approach to simulate the transient response of the fluid to the actuator will be developed.

\section{Acknowledgment}

The present effort was supported by NASA.

\section{References}

1. W.Shyy, B.Jayaraman and A.Anderson, "Modeling of Glow-Discharge Induced Flow Dynamics", J. Appl. Phys., 92(11), pp 6434-6443, 2002.

2. J.R.Roth, D.M.Sherman and S.P. Wilkinson, "Boundary layer flow control with a one atmosphere uniform glow discharge", AIAA paper $98-0328,36^{\text {th }}$ AIAA Aeropsace Sciences Meeting, January 12 15, 1998, Reno NV.

3. J.R.Roth, H.Sin, R.C.M.Madhan and S.P.Wilkinson, "Flow Re-attachment and Acceleration by Paraelectric and Peristaltic Electrohydrodynamic Effects", AIAA paper 2003-0351, $41^{\text {st }}$ Aerospace Sciences Meet \& Exhibit, Reno, NV, Jan 6-9, 2003.

4. J.R.Roth, "Electrohydrodynamically Induced Airflow in a One Atmosphere Uniform Glow Discharge Surface Plasma", Paper 6P-67, Proc. 25th IEEE International Conference on Plasma Science, Raleigh, NC June 1-4, 1998, ISBN 0-7803-4792-7, p 291.

5. C.L.Enloe, T.E.Mclaughlin, R.D.Van Dyken and K.D. Kachner, "Mechanisms and responses of a single dielectric barrier plasma actuator: Plasma Morphology", AIAA J. 42(3), 2004.

6. C.L.Enloe, T.E.Mclaughlin, R.D.Van Dyken, K.D. Kachner, E.J.Jumper, T.C.Corke, M.Post and O.Haddad "Mechanisms and responses of a single dielectric barrier plasma actuator: Geometric effects", AIAA J. 42(3), 2004.

7. C.L.Enloe, T.E.Mclaughlin, R.D.Van Dyken and J.C.Fischer, "Plasma structure in the aerodynamic plasma actuator", AIAA paper 2004-0844, 42 ${ }^{\text {nd }}$ Aerospace Sciences Meet \& Exhibit, Reno, NV, Jan 5$8,2004$.

8. T.C.Corke and E.Matlis, "Phased Plasma Arrays for Unstaeady Flow Control", AIAA paper 20002323, Fluids 2000, June 19-22, 2000, Denver, CO.

9. T.C.Corke, E.J.Jumper, M.L.Post, D.Orlov and T.E.Mclaughlin, "Application of Weakly-ionized Plasmas as Wing Flow-Control Devices", AIAA paper 2002-0350, $41^{\text {st }}$ Aerospace Sciences Meeting \& Exhibit, Reno, NV, Jan 6-9, 2003.

10. R.Van Dyken, T.E.Mclaughlin and C.L.Enloe, "Parametric investigations of a single dielectric barrier plasma actuator", AIAA paper 2004-846, $42^{\text {nd }}$ Aerospace Sciences Meet \& Exhibit, Reno, NV, Jan 5$8,2004$.

11. M.L.Post and T.C.Corke, "Seperation Control on High Angle of Attack Airfoil Using Plasma Actuators", AIAA paper 2003-1024, $41^{\text {st }}$ Aerospace Sciences Meet \& Exhibit, Reno, NV, Jan 6-9, 2003.

12. J.List, A.R.Byerly, T.E.Mclaughlin and R.D.Vandyken, "Using Plasma Actuator to control Laminar Separation on a Linear Cascade Turbine Blade", AIAA paper 2003-1026, $41^{\text {st }}$ Aerospace Sciences Meet \& Exhibit, Reno, NV, Jan 6-9, 2003.

13. B.L.Smith and A.Glezer, "The Formation and Evolutionof Synthetic Jets," Physics of Fluids, Vol. 10, No. 9, 1998, pp. 2281-2297. 
14. R.Rivir, A.White, C.Carter, B.Ganguly, A.Forelines and J.Crafton, "Turbine Flow Control, Plasma Flows", AIAA paper 2003-6055, 41 ${ }^{\text {st }}$ Aerospace Sciences Meet \& Exhibit, Reno, NV, Jan 6-9, 2003.

15. R.Rivir, A.White, C.Carter, B.Ganguly, J.Jacob, A.Forelines and J.Crafton, "AC and Pulsed Plasma Flow Control”, AIAA paper 2004-0847, $42^{\text {nd }}$ Aerospace Sciences Meet \& Exhibit, Reno, NV, Jan 5-8, 2004.

16. S.Leonov, V.Bityurin and D.Yarantsev, "The Effect of Plasma Induced Separation", AIAA paper 2003-3853, 34 ${ }^{\text {th }}$ AIAA Plasmadynamics and Lasers Conference, Orlando, FL, Jun 23-26, 2003.

17. J.S.Shang, "Recent research in magneto-aerodynamics”, Prog. Aero. Sci. , 37, pp. 1-20, 2001

18. J.L.Richardson, A.K. Carr and J.R.Roth, "Increasing the surface energy of materials with a OAUGDP”, Proceedings of ICOPS meeting, May 19-22, San Diego, California, pp 229-230 (1997)

19. J.R.Roth, Principles Vol. I-Principles, Industrial plasma Engineering (IOP, Bristol, 1995).

20. J.R.Roth, Principles Vol. II- Applications to Non-thermal plasma processing , Industrial plasma Engineering (IOP, Bristol, 2001).

21. S.Chan, "The application of plasma and electrohydrodynamic control to subsonic cavity flows", Personal communication. 2004.

22. A.Soldati and S.Banerjee, "Turbulence Modification by Large-scale Organized Electrohydrodynamic flows", Phys. of Fluids, 10(7), pp 1742-1756, 1998.

23. A.Soldati and C.Marchioli, "Prospects for Modulation of Turbulent Boundary Layer by EHD flows", Turbulence Structure and Modulation, Springer-Verlag, Wien, pp. 119-160, 2001.

24. S.P.Wilkinson, "Investigation of an Oscillating Surface Plasma for Turbulent Drag Reduction," AIAA paper 2003-2199, 41 ${ }^{\text {st }}$ Aerospace Sciences Meet \& Exhibit, Reno, NV, Jan 6-9, 2003.

25. M.R.Dhanak and C.Si, "On reduction of turbulent wall friction through spanwise wall oscillations", J. Fluid Mech., 383, pp 175-195, 1999.

26. Y.Du, V.Symeonidis and G.E.Karniadakis, "Drag reduction in wall-bounded turbulence via a transverse traveling wave", J. Fluid Mech., 457, pp 1-34, 2002.

27. T.E.Nitschke and D.B.Graves, "A comparison of particle in cell and fluid model simulations of lowpressure radio frequency discharges”, J. Appl. Phys. 76(10), pp. 5646-5660, 1994.

28. M.J.Kushner, IEEE Trans. Plasma Sci., 14(4), pp. 188, 1986

29. M.J.Kushner, "Monte-Carlo simulation of electron properties in rf parallel plate capacitively coupled discharges", J. Appl. Phys., 54(9), pp. 4958, 1983.

30. D.Vender and R.W.Boswell, "Numerical modeling of low-pressure RF plasmas", IEEE Trans. Plasma Sci., 18(4), pp. 725-732, 1990

31. R.W.Boswell and I.J.Morey, "Self-consistent simulation of a parallel-plate rf discharge", Appl. Phys. Lett. 52(1), pp. 21-23, 1988.

32. M.E.Riley, K.E.Greenberg, G.A.Hebner and P.Drallos, "Theoretical and experimental study of lowtemperature, capacitiviley coupled, radio-frequency helium plasmas”, J. Appl. Phys, 75(6), pp. 27892798, 1994 
33. W.Nicholas G.Hitchon, T.J.Sommerer and J.E Lawler, "A self-consistent kinetic plasma model with rapid convergence”, IEEE Trans. Plasma Sci., 19(2), pp. 113-121, 1991

34. C.K.Birdsall, "Particle-in-cell charged-particle simulations, plus Monte Carlo collisions with neutral atoms", PIC-MCC", IEEE Trans. Plasma Sci., 19(2), pp. 65-85, 1991

35. M.Surendra and D.B.Graves, "Particle simulations of radio-frequency glow discharges", IEEE Trans. Plasma Sci., 19(2), pp. 144-157, 1991

36. M.Surendra, D.B.Graves and G.M.Jellum, "Self-consistent model of a direct-current glow discharge: Treatment of fast electrons", Phys. Rev. A, 41(2), pp. 1112-1126, 1990.

37. F.Massines, R.Ben Gadri, A.Rabehi, Ph.Decomps, P.Segur and Ch.Mayoux, "Experimental and Theoretical Study of a Glow Discharge at Atmospheric Pressure Controlled by Dielectric Barrier", J. Appl. Phys, 83(6), pp 2950-2957, 1998.

38. R.Ben Gadri, "One Atmospheric Glow Discharge Structure Revealed by Computer Modeling," IEEE Trans. Plasma Sci., 27(1), 1999.

39. U.Kogelschatz, B.Eliasson and W.Egli, Pure. Appl. Chem., 71(10), 1819-1828 (1999).

40. Y.B.Golubovskii, V.A Maiorov, J.Behnke and J.F.Behnke, "Modeling of homogeneous barrier discharge in helium at atmospheric pressure", J.Phys. D: Appl. Phys. 36, pp. 39-49, 2003

41. X.Xu and M.J.Kushner, "Multiple microdischarges dynamics in dielectric barrier discharges", J. Appl. Phys. 84(8), pp. 4153-4160.

42. P.Segur and F.Massines, "The role of numerical modeling to understand the behavior and to predict the existence of an atmospheric pressure glow discharge controlled by a dielectric barrier", Proc. Of the XIII $^{\text {th }}$ Int. Conf. of Gas Discharges and their applications, Glasgow, Sep. 3-8, pp. 15-24, 2000.

43. J.P.Boeuf and L.C.Pitchford, "Two-dimensional model of a capacitively coupled $\mathrm{rf}$ discharge and comparisons with experiments in the gaseous electronics conference reference reactor", Phys. Rev. E, 51(2), pp. 1376-1390, 1995.

44. E.P.Hammond, K.Mahesh and P.Moin, "A Numerical method to simulate radio-frequency plasma discharges", J.Comp. Phys., 176, pp 402-429, 2002

45. P.Colella, M.R.Dorr and D.D.Wake, "Numerical solution of plasma fluid equations using locally refined grids", J. Comp. Phys. 152, pp. 550-583, 1999.

46. P.Colella, M.R.Dorr and D.D.Wake, "A conservative finite difference method for the numerical solution of plasma fluid equations”, J. Comp. Phys. 149, pp. 168-193, 1999.

47. D.Lee, J.M.Park and Y.Kim, "Discharge mode transition simulation of dielectric barrier discharge in helium-oxygen mixture", private communication.

48. D.V.Gaitonde, M.R.Visbal and S.Roy, "Control of flow past a wing section with Plasma-based Body forces", AIAA paper 2005-5302, 36 ${ }^{\text {th }}$ Plasma Dynamics And Lasers Conference, 6-9 June, 2005, Toronto.

49. S.Roy and D.V.Gaitonde, "Modeling surface discharge effects of atmospheric RF on gas flow control", ", AIAA paper 2005-0160, $43^{\text {rd }}$ AIAA Aeropsace Sciences Meeting, January 10-13, 2005, Reno NV. 
50. S.Roy and D.V.Gaitonde, "Multidimensional collisional dielectric barrier discharge for flow separation control at atmospheric pressures", AIAA paper 2005-4631, 35 ${ }^{\text {th }}$ AIAA Fluid dynamics conference , 6-9 June, 2005, Toronto, Canada.

51. K.P.Singh and S.Roy, "Simulation of an asymmetric single dielectric barrier plasma actuator", J. App. Phys. 98, 083303-1, 2005.

52. H.Kumar and S.Roy, "Multidimenisonal hydrodynamic plasma-wall model for collisional plasma discharges with and without magnetic-field effects”, Phys. Of Plasmas, 12, 093508, 2005.

53. H.N.Najm, P.S.Wyckoff and O.M. Knio, "A Semi-implicit Numerical Scheme for Reacting Flow-I", J. Comp. Phys. 143, pp. 381-402, 1998.

54. O.M.Knio, H.N.Najm and P.S.Wyckoff, "A Semi-implicit Numerical Scheme for Reacting Flow II", J.Comp. Phys., 154, pp.428-467, 1999.

55. J.G.Verwer, "Explicit Runge-Kutta methods for parabolic partial differential equations", Appl. Numer. Math. 22, pp. 359-379, 1996.

56. P.N.Brown, G.D.Byrne, and A.C.Hindmarsh, "VODE: A variable coefficient ODE solver", SIAM J. Sci. Stat. Comput. 10, pp. 1038, 1989.

57. W.Shyy, S.Thakur, H.Ouyang, J.Liu and E.Blosch, "Computaional Techniques for Complex Transport Phenomena" ( Cambridge university press, UK), 1997

58. W.Shyy, "Computational Modeling For Fluid flow and Interfacial Transport", Elsevier, Amsterdam, 1994.

59. S.Thakur, J.Wright and W.Shyy, "STREAM: A computational fluid dynamics and heat transfer Navier-Stokes solver: theory and applications". Gainesville, FL: Streamline Numerics, Inc; 2002

60. D.A.Knoll and D.E.Keyes, "Jacobian-free Newton Krylov Methods: a Survey of Approaches and Applications", J. Comp. Phys. 193, 357-397, 2004.

61. R.Tyson, L.G.Stern and R.J.Leveque, "Fractional steps method applied to chemotaxis model", J. Math. Biol. 41, 455-475, 2000.

62. B.Sportissee, "An analysis of operator splitting techniques in the stiff case", J. Comp. Phys. 161, 140$168,2000$.

63. S.D.Cohen, A.C.Hindmarsh, CVODE, a stiff/nonstiff ODE solver in C, Comp.Phys. 10 (2) (1996) 138143. 